\title{
AS PRÁTICAS DE EDUCAÇ̃̃̃O EM DIABETES VIVENCIADAS NO SUS: UMA DISEUSSÃO DA LITERATURA COM ÊNFASE NA ATENCẼ̃O PRIMÁRIA À SAÚDE
}

\author{
Luana de Oliveira Souza ${ }^{1}$ \\ Wagner dos Santos Figueiredo², Maria Lúcia Teixeira Machado ${ }^{3}$
}

The education practices in diabetes experienced in Sus: a literature diseussion with emphasis on Primary Health Care

\begin{abstract}
RESUMO
O objetivo deste estudo foi apresentar e discutir as práticas de educação em saúde direcionadas aos portadores de diabetes produzidas no Brasil a partir da criação do SUS, com ênfase na Atenção Primária à Saúde. Tratouse de uma pesquisa bibliográfica e foram consultadas as principais bases de dados em saúde com interseções dos conjuntos dos termos: "educação em saúde", "diabetes" e "Brasil". Foram selecionados 22 trabalhos realizados em âmbito nacional, sendo 18 na Atenção Básica e 4 em outros níveis de atenção. Foram encontradas tanto práticas coletivas, como a educação em grupos, quanto individuais, como consultas, visitas domiciliares e monitoramento telefônico. Uma maior diversidade de formatos de práticas educativas foi identificada na Atenção Básica. Predominaram atividades multiprofissionais realizadas com idosos e que enfatizaram os temas: alimentação saudável, exercício físico e medicamentos. A maior parte das vivências baseou-se na relação dialógica e na abordagem educacional construtivista/problematizadora, com tendência voltada à concepção pedagógica de Paulo Freire. O grande desafio da educação em saúde está vinculado à formação profissional e ao processo de trabalho, portanto é essencial o papel de gestores de saúde no estímulo à capacitação dos profissionais para que possam desenvolver e ampliar habilidades de escuta e linguagem em futuras práticas educativas.
\end{abstract}

PALAVRAS-CHAVE: Educação em Saúde; Diabetes Mellitus; Autocuidado; Atenção Primária à Saúde; SUS.

\begin{abstract}
The objective of this research was to present the practices of health education directed to patients with diabetes produced in Brazil since the SUS creation, with emphasis on the primary health care. The methodology used was the bibliographic survey, and health databases using intersections of terms have been searched: "health education", "diabetes" and "Brazil", selecting just national research. A total of 22 studies describing educational processes was selected, 18 in Primary Care and 4 in other levels of care, resulting in collective educational practices, as group education, and individualized practices, as consultations, home visits, and telephone monitoring. A greater diversity of educational practices formats was seen in primary care. Multidisciplinary activities in which the audience were elderly predominated, discussing the subjects: healthy eating, exercise, and medications. Most of the experiences, both in primary care as in other levels were based on dialogic relationship and constructivist/ problem-based educational approach, with tendencies toward the pedagogical conception of Paulo Freire. The major challenge in health education is related to vocational training and work process, so it is essential that health managers encourage professional training so they can develop and extend listening and language skills.
\end{abstract}

KEYWORDS: Health Education; Diabetes Mellitus; Self-Care; Primary Health Care; SUS.

\footnotetext{
1 Centro de Ciências Biológicas e da Saúde, Programa de Pós-Graduação em Gestão da Clínica, Universidade Federal de São Carlos. E-mail: luli_os@yahoo. com.br.

2 Professor Adjunto do Departamento de Medicina, Programa de Pós-Graduação em Gestão da Clínica, Universidade Federal de São Carlos.

3 Professora Associada do Departamento de Enfermagem, Programa de Pós-Graduação em Gestão da Clínica, Universidade Federal de São Carlos.
} 


\section{INTRODUÇÃO}

No Brasil e no mundo as doenças crônicas não transmissíveis (DCNT) estão cada vez mais prevalentes, afetando a todos os países e classes sociais. O tratamento envolve mudanças de estilo de vida, em um processo de cuidado contínuo que, usualmente, não leva à cura mas sim a um controle da doença. ${ }^{1}$

Entre elas ressalta-se o diabetes mellitus (DM), uma síndrome de etiologia múltipla, decorrente da falta de insulina e/ou da incapacidade de ela exercer adequadamente seus efeitos. Bem como as outras DCNT, o DM é considerado um importante problema de saúde pública mundial devido a sua incidência e prevalência aumentadas em proporções epidêmicas nas últimas décadas. Em 2012 estimava-se que a doença atingia pelo menos 7,4\% dos adultos brasileiros, e esse número vem crescendo em escala exponencial. ${ }^{2}$

O diabetes é uma doença associada a complicações que comprometem a qualidade de vida dos indivíduos, principalmente por causar disfunções e insuficiência de órgãos importantes como olhos, rins, nervos, cérebro, coração e vasos sanguíneos. ${ }^{3}$ Portanto, representa um desafio para os serviços de saúde e merece atenção especial por ser fator de risco para doenças cardiovasculares, principal causa de morte no Brasil.

Quando se realiza uma análise epidemiológica, social e econômica do diabetes, fica evidente a necessidade de políticas públicas de saúde que minimizem as dificuldades que os portadores de diabetes vivem em seu cotidiano, bem como as de seus familiares e da própria sociedade em lidar com a doença. Entre essas políticas, destacam-se aquelas que valorizam ações de incentivo ao autocuidado. ${ }^{4}$ O resultado mais positivo no controle do DM se dá pelo controle da glicemia a partir do desenvolvimento do autocuidado, que contribui para uma melhor qualidade de vida e redução da morbimortalidade, e, portanto, deve ser considerado no acompanhamento dos pacientes nos serviços de saúde. $^{5}$

Com relação à saúde pública brasileira, há anos existem propostas de reorganização da atenção ao DM no Sistema Único de Saúde (SUS), com vistas a aprimorar os serviços prestados dentro das Redes de Atenção à Saúde (RAS), tornando o cuidado mais resolutivo e de qualida$\mathrm{de}^{6}$. Recomenda-se que $60 \%$ a $80 \%$ dos casos de diabetes sejam tratados na Atenção Básica (AB), também chamada de Atenção Primária à Saúde (APS), que é a porta de entrada preferencial do SUS e local privilegiado para os cuidados nas DCNT. ${ }^{7}$

São inúmeras as evidências de que a APS é o melhor modelo de organização dos serviços de saúde, principal- mente quando se trata de DCNT. ${ }^{8}$ As propostas de reorganização do SUS envolvem, principalmente, a atualização dos profissionais de saúde que atuam na $\mathrm{AB}, \mathrm{com}$ foco em estratégias de educação em saúde para o autocuidado e na formação de vínculo com os pacientes. ${ }^{9}$ A última versão da Política Nacional de Atenção Básica reforça a importância da educação em saúde como fator essencial no fortalecimento dos cuidados em DM no âmbito da saúde coletiva. ${ }^{10}$ Ela é capaz de incentivar o diabético a criar maneiras de lidar melhor com a doença e diminuir o índice de complicações. Educação não como mera instrução, mas como via de desenvolvimento de uma comunicação eficaz, que incentive práticas de autocuidado diárias e mudanças de hábitos de vida. A partir de escolhas adequadas, associadas a um amplo apoio das instituições de saúde, família e comunidade, o diabético pode ter melhores condições de assumir o controle de sua condição crônica. ${ }^{4}$

Atualmente já se sabe que as práticas educativas tradicionais focadas na transmissão de conhecimento do profissional de saúde para o paciente não dão mais conta da complexidade do autocuidado no diabetes. ${ }^{11}$ Muitos estudos propõem práticas educativas baseadas principalmente no referencial teórico-filosófico de Paulo Freire, o qual não considera o educador como detentor do conhecimento. Segundo o pensamento freiriano, conhecimento não é algo pronto, mas sim algo construído a partir daquilo que é significativo para cada indivíduo, a partir do diálogo e troca de experiências. Preza-se uma relação horizontal entre os sujeitos, onde cada um assume uma posição ativa frente aos seus cuidados em saúde. ${ }^{12,13}$

Foi dentro desse contexto de educação em saúde como parte dos cuidados aos diabéticos que essa pesquisa se desenvolveu. O objetivo central foi identificar e discutir as práticas de educação em saúde direcionadas aos portadores de diabetes apresentadas na literatura científica produzida no Brasil desde a criação do SUS, com ênfase na APS, por ser espaço privilegiado para práticas educativas.

\section{MATERIAIS E MÉTODOS}

Foi utilizada a metodologia da pesquisa bibliográfica para identificar as práticas de educação em saúde direcionadas aos portadores de diabetes, como forma de garantir que fossem coletadas e discutidas tanto evidências científicas na literatura quanto material empírico e experiências sobre as práticas educativas. Teve-se a intenção de debater o que já foi produzido sobre o tema, fator fundamental para impulsionar o aprendizado e guiar o cuidado na prática em saúde ao portador de diabetes.

Para realizar as buscas de artigos foram utilizadas as bases de dados LILACS, MEDLINE, Biblioteca Cochra- 
ne e SciELO e teses e dissertações selecionadas no Banco de teses da CAPES (Coordenação de Aperfeiçoamento de Pessoal de Nível Superior) disponível em http://bancodeteses.capes.gov.br/. Os descritores utilizados nas bases de dados foram: "educação em saúde", "diabetes" e "Brasil", bem como seus termos similares em língua inglesa, utilizados de forma combinada para encontrar trabalhos que contivessem esses termos no título, descritores, resumo ou assunto.

Foram incluídos estudos que descreveram processos educacionais em saúde voltados aos portadores de diabetes desde a criação do SUS, tanto na APS quanto em outros níveis de atenção à saúde, permitindo, assim, que se fizesse uma reflexão sobre as divergências e semelhanças entre as práticas educativas realizadas nos diferentes níveis de atenção à saúde. Foram selecionados somente trabalhos com acesso ao texto completo nas bases de dados, realizados em âmbito nacional no SUS. Incluíram-se estudos que tinham como público-alvo pessoas portadoras de qualquer tipo de diabetes e de todas as faixas etárias, bem como em qualquer etapa da doença. Foram excluídos os estudos que não se enquadraram nos critérios citados.

Realizaram-se pesquisas periódicas nas bases de dados para garantir a ausência de repetições e a inclusão de novos estudos que pudessem surgir durante o prazo de busca, que foi de junho a novembro de 2014. Os estudos que alcançaram os critérios foram selecionados para a amostra bibliográfica e os dados foram compilados em um banco de dados no software Microsoft Excel. ${ }^{\circledR}$

Foi realizada uma leitura crítica e interpretativa do material selecionado, com a necessária imparcialidade e objetividade, buscando respostas aos objetivos do estudo. Esta pesquisa é resultado de dissertação de mestrado e não foi financiada. Não houve conflitos de interesse e não foi necessário envio a Comitê de Ética por não envolver participação direta de seres humanos, portanto não implicou em riscos ou prejuízos a indivíduos.

\section{RESULTADOS E DISCUSSÃO}

Foram selecionados 14 artigos publicados entre $2002 \mathrm{e}$ 2014, sendo que a base de dados que mais resgatou artigos foi a Scielo. Entre eles, 11 descrevem práticas de educação entre relatos de experiência, estudos de caso e propostas de intervenção, e 3 são revisões da literatura sobre o tema. Já entre as teses e dissertações, 8 foram selecionadas para este trabalho, sendo somente 1 revisão e, as outras, estudos de abordagem quantitativa e/ou qualitativa, publicados de 2011 em diante devido à disponibilidade do Banco de Teses da CAPES.

Como primeira reflexão da busca na literatura, é possí- vel citar que, apesar de ser reconhecida internacionalmente a importância de programas educativos para promover melhor qualidade de vida e o autocuidado, poucas pesquisas descrevem esse tipo de intervenção no SUS, o que vai ao encontro do relatado em um dos estudos ${ }^{14}$. Ademais, foram encontrados somente trabalhos publicados a partir de 2002, reforçando a falta de publicações principalmente nas primeiras décadas do SUS.

Segundo os autores incluídos nesta revisão, existem diversas definições para o tema "educação em saúde", classificada como estratégia, plano, ferramenta ou proposta. A maioria dos autores concorda que é um caminho eficaz para que o diabético se conscientize sobre a importância do autocuidado e da mudança no estilo de vida, a partir da discussão entre usuários e profissionais de saúde sobre as informações e da reflexão sobre a doença e seu tratamento. O estímulo ao autocuidado vindo dos profissionais facilita o compromisso dos diabéticos com ações que visem seu bem-estar, promovendo melhor qualidade de vida.

Rezende $(2011)^{15}$ cita que a educação em saúde deve ser entendida não como uma simples ação, mas como um processo que auxilia o diabético e ter melhores atitudes a partir do aproveitamento de experiências formais e informais. $\mathrm{O}$ autor enfatiza que os processos educativos são a chave para as intervenções preventivas no âmbito coletivo, principalmente aqueles nos quais profissionais de saúde e pacientes compartilham conhecimentos de forma ativa e corresponsável. A seguir será feito um desdobramento das principais características encontradas nas práticas educativas.

\section{Características das práticas educativas}

\subsection{Público-alvo}

A maioria dos estudos abordou propostas de educação tendo como público-alvo somente diabéticos, porém alguns deles propuseram práticas educativas para hipertensos e diabéticos conjuntamente. Ações educativas que abordam mais de um agravo à saúde podem ser eficazes por tratarem o indivíduo não como uma patologia, mas sim como uma pessoa, com toda a sua complexidade e subjetividade. Frequentemente, as doenças crônicas estão relacionadas aos hábitos de vida e acometem os mesmos indivíduos, devendo ser cuidadas e trabalhadas em conjunto. ${ }^{7,9}$

O número total de participantes inseridos nas ações educativas foi bem variado, a depender da atividade. Ações grupais envolveram mais participantes, subdivididos em grupos menores a cada encontro. A forma de recrutamento e seleção dos participantes variou entre de- 
manda espontânea, encaminhamento pela equipe e convite a indivíduos cadastrados nos serviços de saúde, com predominância de participação de pacientes já em seguimento na rotina do serviço.

A faixa-etária predominante foi superior a 60 anos, mostrando incidência maior de práticas educativas voltadas a idosos, focadas principalmente no DM tipo 2, a qual acomete indivíduos adultos e idosos e tem uma alta prevalência se comparada com outros tipos de diabetes. Nenhum estudo teve como público-alvo especificamente diabéticos tipo 1. Esses achados corroboram a revisão de Borba (2012), ${ }^{16}$ que observou uma predominância de faixas etárias mais avançadas e apontou para a necessidade de pesquisas que planejem práticas educativas para idosos, por ser grupo de risco para as DCNT e pela dificuldade de adaptação às modificações exigidas para o controle metabólico.

\subsection{Cenário das práticas educativas}

Todos os níveis de atenção à saúde foram contemplados, incluindo práticas hospitalares, ambulatoriais e a nível primário. A maioria dos trabalhos foi desenvolvida na APS em UBS tradicional, com poucos estudos em Unidades de Saúde da Família (USF). Esses achados estão de acordo com os relatos da revisão de Borba (2012), ${ }^{16}$ a qual encontrou, entre os estudos revisados, todos os níveis de atenção à saúde como cenários de práticas educativas no Brasil, com predominância da APS.

Para além do espaço físico das unidades de saúde, alguns estudos relataram atividades educativas realizadas em equipamentos sociais do território de abrangência ou dentro do domićlio dos usuários. Torres, Reis, Roque e Faria (2013, p. 97) ${ }^{17}$ citam que "a maior parte da educação do autocuidado em diabetes ocorre fora do serviço de saúde, enquanto as pessoas estão vivendo sua vida em casa e na comunidade". Além de abranger uma gama maior de diabéticos que não procuram o serviço ou têm dificuldades físicas para chegar até o local, essas atividades podem incentivar atitudes positivas nos diabéticos, levando em conta seu próprio ambiente e contexto de vida.

Conclusivamente, a APS demonstrou-se como o nível de atenção com maior potencialidade para enfrentar os problemas por meio da educação em saúde. O vínculo, princípio da APS, auxilia na concretização das ações de saúde nas DCNT e é um diferencial entre esse e os outros níveis de atenção. ${ }^{18}$

\subsection{Modos de fazer a educação em saúde}

As publicações oficiais do Ministério da Saúde estimu- lam os trabalhadores de saúde a realizar ações educativas com formatos que incentivem a participação do usuário, como campanhas educativas, grupos e ações comunitárias para promover estilo de vida saudável, na tentativa de ultrapassar estratégias repressivas como palestras e orientações impositivas. No entanto, os relatos da literatura mostram que prevalecem atividades em que a voz do profissional de saúde é o foco principal. ${ }^{19}$

Nesta revisão foram encontradas diversos formatos de práticas educativas, tanto coletivas quanto individuais. Grupos, consultas individuais, visitas domiciliares e contato via telefone foram as ações citadas. Todas as atividades em nível ambulatorial foram exclusivamente no formato de grupos, já as ações realizadas na $A B$ foram variadas, e, em sua maioria, utilizaram mais de uma estratégia educativa, paralelamente ou uma dando continuidade à outra. Esse dado confirma a abrangência de cuidados que se pode oferecer na $A B$, espaço privilegiado para as mais diversas formas de ação educativa no DM.

O objetivo da maioria das atividades foi orientar, discutir informações e transmitir conteúdos programáticos, mas também foram citados objetivos paralelos, como sensibilizar para o autocuidado, aferir sinais vitais e glicemia, promover o aconselhamento nutricional, praticar exercício físico, realizar avaliação antropométrica e dispensar medicação.

Entre as práticas grupais, a maioria se subdividiu em encontros de acordo com os temas abordados. Houve predomínio de atividades semanais com duração de 1 a 2 horas cada. Segundo autores, ${ }^{20,21}$ quando os encontros acontecem com intervalo menor, os participantes tendem a faltar menos, estreitar relações e se sentirem mais motivados. No estudo de Vieira (2011), ${ }^{22}$ grande parte dos usuários considerou o tempo dos encontros insuficiente e desejou que fossem mais longos, referindo ter sido prazeroso estar nos grupos pela troca de experiências e possibilidade de serem escutados.

A educação em saúde para as DCNT praticada em grupo é uma prática assistencial secular, principalmente na $\mathrm{AB} \cdot{ }^{15} \mathrm{Na}$ concepção dos próprios diabéticos, os grupos funcionam como espaço em que eles são acompanhados e orientados para o autocuidado. Para além de receber orientações, Silva, Costa e Fermino $(2008)^{23}$ relataram o grupo como "uma perspectiva no atendimento às pessoas que viabiliza a troca de experiências no intuito de promover aprendizagem, respeitando a cultura e a história dos participantes".

A dinâmica de grupo estimula o convívio social e as ligações afetivas entre os diabéticos e os profissionais de saúde, facilitando a discussão entre sujeitos com mesmos objetivos. Essa interação pode auxiliar no entendimento 
da doença, com toda sua complexidade, e na quebra de barreiras, permitindo aos usuários expressarem suas dúvidas e expectativas, além do apoio mútuo e sugestões construtivas de quem vivencia ou já vivenciou as mesmas dificuldades. Como consequência, há acréscimo no conhecimento grupal e individual, o qual pode acarretar atitudes de autocuidado e mudança de hábitos de vida. ${ }^{16,20,23,24}$

Apesar da importância dos grupos, alguns autores ${ }^{25,26}$ referiram dificuldades para realizá-los devido à baixa adesão. Ambos os trabalhos referidos foram realizados na $\mathrm{AB}$, em UBS com Equipes de Saúde da Família. Mendonça ${ }^{26}$ relaciona essa baixa adesão ao desconhecimento da importância desse tipo de estratégia por parte da população, além do interesse maior por consultas médicas individuais. Segundo o autor, a população foi acostumada a uma prática restritiva ao longo de décadas, baseada em consultas e solicitação de exames, sendo, portanto, comum perceber atitude de resistência diante de práticas que fujam daquilo a que está adaptada. Prestar somente assistência individual pode acarretar no aumento da demanda do serviço por preencher as agendas dos profissionais e na restrição da assistência prestada ao diabético.

A revisão proposta por Grillo (2011) ${ }^{27}$ comparou a educação individual e aquela realizada em grupos com relação à melhora dos exames clínicos e não encontrou diferença significativa, porém cita a educação em grupo como mais viável por abranger maior número de pessoas. Por outro lado, o grupo não deve ser a única estratégia no controle das DCNT devido aos impactos sociais, econômicos e emocionais dessas doenças, sendo ideal que se programe um amplo conjunto de ações educativas na assistência aos portadores de DM. Nesse sentido, é essencial que as equipes se dediquem a construir ações programadas de prevenção e promoção à saúde, dialogando com a população para construir espaços coletivos que atendam suas expectativas. ${ }^{14,26,28,29}$

O contato telefônico mostrou-se uma opção de comunicação viável e efetiva para o monitoramento dos usuários com diabetes por ser simples, cômodo, rápido e de baixo custo. O telefone encurta distâncias físicas e socioculturais, fortalecendo o vínculo e facilitando a escuta e compreensão, principalmente quando se trata da APS. Os usuários ficam satisfeitos ao receber um telefonema, pois se sentem acolhidos e motivados, portanto pode isso ser encarado como uma forma de reforço de um plano educativo e garantia da longitudinalidade do cuidado. ${ }^{17,18}$

As visitas domiciliares também se mostraram um método educativo efetivo, por possibilitarem a compreensão, por parte dos profissionais de saúde da APS, do contexto em que diabéticos e suas famílias vivem e dos recursos de que dispõem. É um momento de aproximação com o usuário e sua realidade, no qual é possível fortalecer o vínculo e oferecer uma singularidade no cuidado. Pode ser uma estratégia vantajosa por quebrar obstáculos de acesso aos serviços, como distância de moradia, limitação física ou dificuldades com custos de deslocamento. ${ }^{25,30,31}$

Os relatos dos trabalhos incluídos nesta revisão indicam a diversidade de formatos de práticas educativas existentes na literatura. Fica nítido que é necessário um conjunto de práticas educativas nos cuidados com os diabéticos, principalmente na APS, levando em conta as necessidades de cada comunidade, para desenvolvê-las de forma a incentivar a coparticipação.

\subsection{Profissionais envolvidos}

A maior parte das práticas educativas incluídas nesta revisão foi conduzida por equipe multiprofissional, tanto ambulatorialmente quanto na APS. Houve grande diversidade de profissionais, a citar: fisioterapeuta, profissional de educação física, terapeuta ocupacional, agente comunitário de saúde, farmacêutico, psicólogo, assistente social, fonoaudiólogo e dentista, porém a participação efetiva foi de profissionais de enfermagem, médicos e nutricionistas, possivelmente por serem especialidades com grande afinidade com os conteúdos abordados.

Vale o destaque dado ao profissional médico em algumas publicações. ${ }^{15,32}$ Rezende $(2011)^{15}$ reforça a valorização desse profissional por parte da população, ainda ligada ao modelo tradicional de assistência à saúde, centralizando o papel de cuidado na figura desse ator.

A revisão realizada por Grillo $(2011)^{27}$ relata que existem poucos estudos na literatura que buscam avaliar se existem diferenças entre as práticas realizadas por indivíduos com diferentes formações profissionais, e, entre elas, o tipo de profissional não parece influenciar nos resultados. Mais do que diferenciar as práticas educativas segundo o núcleo profissional, o trabalho em equipe pode ser mais efetivo no acompanhamento e monitoramento dos diabéticos de um serviço de saúde, evitando a sobrecarga de trabalho. $\mathrm{O}$ fator primordial é a capacitação profissional, principalmente aquela que estimula o indivíduo a desenvolver práticas participativas e supere a perspectiva biológica no processo pedagógico. ${ }^{25,26}$

\subsection{Temas abordados}

Entre os temas abordados nas atividades, destacou-se a tríade do autocuidado: alimentação saudável, exercício físico e medicamentos. A abordagem desses temas é essencial dentro das práticas educativas, por fazerem parte das ações cotidianas necessárias ao bom controle glicêmi- 
co e serem assuntos de interesse entre os próprios diabéticos.

A alimentação é um tema que, quando abordado, necessita de estratégias que considerem os aspectos culturais e regionais do diabético e sua família, pois hábitos alimentares são construídos ao longo de uma vida, com influência sociocultural. ${ }^{16,31}$ Enfatizar a alimentação saudável na prevenção e tratamento das DCNT vai ao encontro das orientações da Política Nacional de Alimentação e Nutrição. ${ }^{33}$

Observou-se uma prevalência de abordagens relacionadas a assuntos e procedimentos médico-farmacológicos, como fisiopatologia, complicações, conceito da doença, fatores de risco, sinais e sintomas, controle glicêmico e insulinoterapia. A valorização desses assuntos tem um aspecto cultural, sendo predominante entre profissionais de saúde e população. ${ }^{26}$

Um número menor de estudos abordou, paralelamente, assuntos ligados aos fatores psicossociais, como controle da ansiedade, facilidades e dificuldades para o autocuidado, hábitos de vida cotidianos e os sentimentos no contexto do DM, como ansiedade, estresse e raiva. Poucos estudos citaram a discussão sobre qualidade de vida e os cuidados com os pés.

$\mathrm{Na}$ maioria dos trabalhos, o enfoque do tema a cada encontro variou em função dos recursos de cada serviço e do profissional responsável pela atividade. A divisão por temas pode facilitar a compreensão dos diabéticos, principalmente entre idosos. Grillo (2011) ${ }^{27}$ apontou para a necessidade de reforço dos temas ao longo do processo educativo, para que os indivíduos possam, após uma primeira discussão, refletir sobre seu autocuidado e, então, concretizar a experiência educativa.

Em suma, mais do que exigir técnicas de algum(ns) profissional(ais) específico(s), as práticas educativas em DM precisam de profissionais que estejam aptos a ouvir as necessidades dos participantes e a trabalhar em equipe. Os temas abordados devem basear-se em temáticas relacionadas aos hábitos de vida saudáveis, porém mais do que um conteúdo pronto tais temas devem ser discutidos levando em conta a demanda do(s) participante(s).

\subsection{As estratégias e metodologias educacionais}

A escolha da concepção pedagógica é fundamental quando se trata de educação em saúde, para propiciar ao usuário a possibilidade de ser crítico e elaborar o conhecimento, pois apenas quando há uma mudança de percepção e processamento da informação é que a aprendizagem torna-se significativa para os sujeitos. ${ }^{29}$ A maior parte das vivências que citou a abordagem educacional utilizada baseou-se na troca de experiências, relação dialógica, educação participativa e abordagem construtivista/problematizadora, com tendência voltada ao pensamento de Paulo Freire. Outro referencial teórico citado foi Dorothea Orem, que se baseia na Teoria do Autocuidado, a qual foca na promoção da autonomia do sujeito, considerando seus valores e motivações.

A preferência pela concepção metodológica de Paulo Freire também foi citada em revisões da literatura. ${ }^{16,34}$ Essa abordagem vai além de apenas repassar conhecimento, oferecendo a oportunidade de percepção e reflexão sobre a situação vivenciada por todos os indivíduos presentes, encaixando-se perfeitamente nas necessidades das práticas de saúde, principalmente quando se trata da $\mathrm{AB}$. A educação com metodologia problematizadora baseada no diálogo tende a promover a quebra da relação verticalizada entre profissional e paciente, aproximando-os e qualificando o cuidado prestado, por adaptar-se às necessidades e realidades dos envolvidos. Além do benefício para o usuário, essa abordagem permite uma reflexão da própria equipe com relação ao seu processo de trabalho. ${ }^{35}$

A revisão bibliográfica de Cyrino, Schraiber e Teixeira (2009) ${ }^{11}$ revela que, nas últimas décadas, tem-se percebido uma ligeira mudança no campo da pesquisa sobre educação em saúde no contexto do diabetes, com estratégias educacionais mais voltadas para o diálogo e a valorização das experiências dos pacientes. Contudo, apesar dos avanços, ainda permanecem muitas dúvidas desafiadoras para a organização de práticas educativas que valorizem a autonomia do sujeito. Nessa revisão ainda foram encontrados estudos ligados à metodologia tradicional, em grupos explicativos ou consultas baseadas na transmissão de conhecimento, mostrando a inadequação com as teorias mais atuais de educação.

É nítida a dificuldade que as equipes ainda têm no lidar com habilidades incentivadoras e participativas, o que pode estar relacionado aos cursos de formação profissional na área da saúde, permeados pela concepção biomédica. Os profissionais tendem a reproduzir tais concepções em sua prática, que, no caso da educação em saúde, se tornam ações patologizantes e verticalizadoras. ${ }^{19}$

Entre os recursos pedagógicos utilizados, o termo prioritariamente citado entre os estudos foi "grupo educativo", mas também foram mencionados grupos operativos, dinâmicas lúdicas, grupos focais, círculo de discussão, roda de conversa e jogos. Esses instrumentos podem auxiliar no processo educativo por contribuir com a criatividade e criar um ambiente prazeroso de aprendizado. ${ }^{20}$

A ferramenta mais citada para condução dos processos educativos em todos os tipos de intervenção foi a palavra. Quando utilizada na forma de palestras ou orien- 
tação unidirecional, em consultas, ela permite pouca ou nenhuma participação ativa dos membros, ${ }^{23,25,28}$ porém quando associa-se a forma verbal a outras ferramentas, a ação torna-se mais efetiva, principalmente nos grupos. Foram citados materiais visuais e de áudio, como imagens, cartazes, figuras, folhetos explicativos, vídeos, teatro e músicas para ilustrar e elucidar os diferentes assuntos. Já nas visitas domiciliares e monitoramento telefônico vale a ênfase para a utilização de roteiro semiestruturado e mapa de conversação.

Independentemente do instrumento escolhido, a maioria das pesquisas se preocupou em utilizar linguagem e materiais adaptados à realidade dos usuários, o que mostra a singularidade das diversas práticas educativas em cada realidade. Utilizar diferentes ferramentas e instrumentos ajustados ao contexto da comunidade pode auxiliar no processo educativo, uma vez que são capazes de estimular o diálogo e ampliar o cuidado.

\subsection{A percepção dos diabéticos: dificuldades e be- nefícios}

As dificuldades enfrentadas pelos diabéticos devem ser levadas em conta no momento de se elaborar um processo educativo. Quando consideradas, podem deixar de ser barreiras e facilitar o aprendizado e a reflexão, gerando resultados benéficos aos participantes.

Segundo os estudos incluídos nesta pesquisa, a maior dificuldade relatada pelos diabéticos esteve relacionada com o baixo nível socioeconômico. A questão financeira pode ser limitante tanto no seguimento de um plano alimentar quanto na realização de exercícios físicos com equipamentos. ${ }^{22}$ As crenças alimentares também dificultam o autocuidado e, comumente, os diabéticos encaram as orientações como uma proibição alimentar. O aconselhamento nutricional é essencial nas ações educativas, porém não de forma impositiva, sendo essencial ter cautela ao orientar um plano e modificar toda uma história de hábitos alimentares. ${ }^{15,22,36}$ Nesse sentido, levar em conta a cultura e crenças sobre alimentação saudável/atividade física e o quanto elas interferem no orçamento familiar facilita a discussão dos temas durante as atividades educativas, as quais se tornam espaços para a oferta de opções mais acessíveis e adaptadas à realidade dos usuários.

Segundo os diabéticos, a baixa escolaridade representa um obstáculo para a compreensão e interpretação das informações, e, assim como a falta de apoio familiar, interfere negativamente no autocuidado. No estudo de Rezende $(2011)^{15}$ relata-se tentativa de mudança de hábitos, porém as "tentações" e pouca disponibilidade de alimentos saudáveis no ambiente familiar foram limitantes. Portanto, o cuidado ao diabético deve envolver todos aqueles com os quais ele convive. ${ }^{22}$

Outros interferentes negativos estiveram relacionados a aspectos psicossociais, como falta de motivação, dificuldade para assumir mudanças, sentimentos negativos, estado emocional, não aceitação da doença, estresse e dificuldade de autocontrole. Sentimentos e emoções podem aflorar no diagnóstico da doença ou nos momentos de adaptação à nova rotina necessária para o bom controle do DM. Expressar e compartilhar sentimentos e dificuldades em grupo pode ser uma atitude incentivadora. ${ }^{17,22,28,31}$ As dificuldades físicas, como dores, problemas articulares e cardíacos também foram consideradas negativas, bem como o acúmulo de trabalho, falta de companhia e de segurança para prática de atividade física.

O principal benefício citado pelos portadores de DM diz respeito ao aumento de conhecimento sobre a doença e tratamento. Conhecimento não necessariamente gera mudança de comportamento, mas é a base para o autocuidado em conjunto com a participação efetiva do diabético. O compartilhamento de experiências, informações e sentimentos também foram fatores positivos resultantes das ações, por serem capazes de estimular a reflexão sobre ser diabético. Também foi citado o benefício do incentivo às mudanças de estilo de vida, pois os diabéticos se sentem motivados para planejar suas refeições, cumprir os horários e praticar exercícios físicos regularmente. Como consequência, há melhor controle glicêmico e metabólico.

Os encontros de educação em saúde foram reconhecidos como espaços para ofertar apoio psicológico, conhecer novas pessoas, fazer amizades e se distrair, o que eleva a autoestima e estimula maior comprometimento, motivação, autonomia e melhora da autopercepção da saúde. Portanto, segundo os próprios diabéticos, as práticas educativas são capazes de estimulá-los a compreender seus problemas e escolher a solução apropriada para o gerenciamento dos cuidados da doença, devendo ser praticadas na rotina dos serviços de saúde.

\subsection{Competências dos profissionais de saúde}

Toda prática educativa exige que os profissionais de saúde tenham habilidades para o planejamento, condução e avaliação das atividades. A característica exigida dos profissionais que mais prevaleceu entre os estudos foi o uso de linguagem clara e objetiva, sendo acessível e simples, adaptada ao sujeito com quem se está lidando. Por serem facilitadores, os profissionais de saúde precisam de linguagem apropriada para aprimorar a comunicação e estimular os participantes a encontrarem estratégias de enfrentamento dos problemas. ${ }^{16}$ 
A capacidade de escuta, de comunicação e a qualidade da interação foram posturas desejadas. A construção de relações dialógicas entre os atores contribui para o vínculo e relação de confiança entre profissionais e pacientes. Ser capaz de compreender e trabalhar os sentimentos dos participantes em um processo educativo tranquiliza e apoia os diabéticos em suas dúvidas e angústias. Para tanto, é necessário captar informações por meio não somente da palavra, mas da entonação da voz, gestos e expressões. ${ }^{17,21,31,35}$

Outra competência citada foi o aprimoramento técnico-científico. O processo de formação profissional deve permitir que desenvolvam habilidades pedagógicas e de compreensão do que está sendo expressado das mais diversas maneiras. Mesmo nos tempos atuais, persiste uma carência de profissionais capazes de estimular uma relação participativa com a população. ${ }^{26}$

Levar em conta as considerações, os valores e motivações, o contexto sociocultural e a realidade de cada indivíduo/família/comunidade foram fatores citados como diferenciais nas ações, pois as tornam mais realistas e efetivas, auxiliando na criação de abordagens singularizadas. O envolvimento direto dos profissionais com o processo educativo, desde o planejamento até a avaliação das atividades, bem como a padronização de condutas entre os diferentes profissionais também foram considerados essenciais. Nos processos educativos grupais, os profissionais de saúde precisam desenvolver a habilidade de trabalhar no modelo educativo participativo, possibilitando maiores trocas e compartilhamentos de informações e percepções. Para tanto, são necessárias estratégias pedagógicas adequadas e o incentivo à autonomia. ${ }^{24,26}$

Algumas características individuais dos profissionais de saúde também foram citadas, como respeito, cautela, persistência, paciência, motivação pessoal, empatia e capacidade de controlar o tempo da atividade educativa, principalmente quando se trabalha com grupos, além da capacidade de considerar o conhecimento popular e a cultura regional. ${ }^{17,20,23}$

A valorização das relações humanas, a prática de atitudes inovadoras e criativas, a consideração dos fatores psicossociais e a capacidade de saber lidar com as dificuldades dos participantes também foram competências exigidas dos profissionais. Cabe ao profissional de saúde mediar o conflito entre as emoções e as ações de autocuidado e estimular o envolvimento da família. ${ }^{20,24,26,29,31}$

Uma postura persistente frente à falta de motivação de cada usuário e a oferta de reforço positivo nas ações de autocuidado podem influenciar positivamente a adesão ao tratamento. ${ }^{26,37}$ É essencial uma equipe que atue de forma interdisciplinar, com forte integração entre os membros da equipe, para que as ações sejam planejadas e desenvolvidas em conjunto e se tornem mais amplas e completas. ${ }^{16}$ Portanto, os serviços de saúde devem contar com profissionais de saúde capacitados tecnicamente para planejar e realizar práticas de educação em saúde adaptadas ao contexto do diabético e de seus familiares, além de desenvolver habilidades de comunicação para trabalhar no modelo participativo.

\section{CONCLUSÃO}

A educação em saúde é uma das estratégias que pode contribuir para reduzir a prevalência de complicações em pessoas com DM, ou minimamente retardá-las, por ser capaz de criar ferramentas de incentivo ao autocuidado. Os resultados deste estudo evidenciaram os benefícios das práticas educativas para os portadores de DM, principalmente na APS, como acréscimo de conhecimento, incentivo à reflexão e mudanças no estilo de vida, troca de experiências e melhora no controle glicêmico.

Existem diferentes maneiras efetivas de promover educação em saúde, como grupos educativos, assistência individual na forma de consultas ou visitas domiciliares e monitoramento telefônico. Nesta revisão, prevaleceram atividades em âmbito coletivo e as práticas grupais foram aquelas que permitiram maior troca de conhecimentos e coparticipação. Todos os serviços de saúde, tanto na $\mathrm{AB}$ como em unidades ambulatoriais, foram cenários de atividades educativas, mas evidenciou-se uma predominância de práticas desenvolvidas na APS, coerente com as políticas públicas que a mostram como cenário privilegiado para ações de educação em saúde.

A participação de equipe multiprofissional mostrouse como diferencial no desenvolvimento adequado dos diversos assuntos relacionados à doença e ao tratamento, levando em conta o contexto e as dificuldades dos diabéticos. $\mathrm{O}$ envolvimento dos profissionais da equipe no processo educativo, com habilidades de comunicação, mostrou-se essencial na formação do vínculo e no estímulo às mudanças de estilo de vida.

Houve diversidade com relação às estratégias educacionais utilizadas entre os estudos incluídos nesta revisão, porém, todos eles reconheceram enfaticamente a importância de novas estratégias de ensino que valorizem o diálogo, a reflexão, a autonomia e a corresponsabilidade dos diabéticos, inspiradas principalmente na concepção de Paulo Freire.

Conclusivamente, o processo de aprendizagem é complexo e sua efetividade depende de diversos fatores relacionados com as equipes de saúde e com o contexto do próprio portador de diabetes. O caminho pode estar 
em assumir a metodologia problematizadora como diretriz para as atividades educativas no DM, principalmente quando se atua na APS. Faz-se necessário que os gestores de saúde incentivem os profissionais para que as futuras práticas educativas estimulem a reflexão entre os usuários, para que esses sejam capazes de atuar diligentemente em seu autocuidado.

\section{REFERÊNCIAS}

1. Ministério da Saúde (Brasil). Portaria n. 483, de $1^{\circ}$ de abril de 2014. Redefine a Rede de Atenção à Saúde das Pessoas com Doenças Crônicas no âmbito do Sistema Único de Saúde (SUS) e estabelece diretrizes para a organização das suas linhas de cuidado. Ministério da Saúde: Gabinete do Ministro. Diário Oficial da União, Brasília, DF, 1 abr 2014. Disponível em: <http://bvsms.saude. gov.br/bvs/saudelegis/gm/2014/prt0483_01_04_2014. html>.

2. Ministério da Saúde (Brasil). Secretaria de Vigilância em Saúde. Departamento de Vigilância de Doenças e Agravos não Transmissíveis e Promoção de Saúde. Vigitel Brasil 2012: vigilância de fatores de risco e proteção para doenças crônicas por inquérito telefônico. Brasília: Ministério da Saúde; 2013.

3. Sociedade Brasileira de Diabetes. Consenso brasileiro sobre diabetes 2002: diagnóstico e classificação do diabetes melito e tratamento do diabetes melito do tipo 2. Rio de Janeiro: Diagraphic; 2003.

4. Ministério da Saúde (Brasil). Estratégia nacional para educação em saúde para o autocuidado em diabetes mellitus. Florianópolis; 2009.

5. Ministério da Saúde (Brasil). Secretaria de Atenção à Saúde. Departamento de Atenção Básica. Estratégias para o cuidado da pessoa com doença crônica: diabetes mellitus. Brasília: Ministério da Saúde; 2013.

6. Ministério da Saúde (Brasil). Secretaria de Atenção à Saúde, Departamento de Atenção Básica. Documento de diretrizes para o cuidado das pessoas com doenças crônicas nas Redes de Atenção à Saúde e nas linhas de cuidado. Brasília: Ministério da Saúde; 2012.

7. Ministério da Saúde (Brasil). Secretaria de Políticas Públicas. Manual de hipertensão arterial e diabetes mellitus. Brasília: Ministério da Saúde; 2002.
8. Ministério da Saúde (Brasil). Secretaria de Atenção à Saúde. Departamento de Atenção Básica. Estratégias para o cuidado da pessoa com doença crônica. Brasília: Ministério da Saúde; 2014.

9. Ministério da Saúde (Brasil). Secretaria de Políticas de Saúde. Departamento de Ações Programáticas Estratégicas. Plano de reorganização da atenção à hipertensão arterial e ao diabetes mellitus. Brasília: Ministério da Saúde; 2001.

10. Ministério da Saúde (Brasil). Secretaria de Atenção à Saúde. Departamento de Atenção Básica. Política Nacional de Atenção Básica. Brasília: Ministério da Saúde; 2012.

11. Cyrino AP, Schraiber LB, Teixeira RR. A educação para o autocuidado no diabetes mellitus tipo 2: da adesão ao "empoderamento". Interface (Botucatu) [Internet]. 2009 set [Citado em: 17 nov 2014];13(30):93-106. Disponível em: <http://www.scielo.br/scielo.php?script=sci_ arttext\&pid $=$ S1414-32832009000300009\&lng $=$ en $>$.

12. Freire P. Conscientização: teoria e prática da libertação, uma introdução ao pensamento de Paulo Freire. 3a. ed. São Paulo: Moraes; 1980.

13. Freire P. Pedagogia da autonomia: saberes necessários à prática educativa. 33a. ed. São Paulo: Paz \& Terra; 2006.

14. Silva TR, Feldmam C, Lima MHA, Nobre MRC, Domingues RZL. Controle de diabetes mellitus e hipertensão arterial com grupos de intervenção educacional e terapêutica em seguimento ambulatorial de uma Unidade Básica de Saúde. Saude Soc [Internet]. 2006 dez [Citado em: 14 nov 2014];15(3):180-9. Disponível em: <http:// www.scielo.br/scielo.php?script $=$ sci_arttext\&pid=S0104$12902006000300015 \& \operatorname{lng}=\mathrm{en}>$.

15. Rezende AMB. Ação educativa na Atenção Básica à Saúde de pessoas com diabetes mellitus e hipertensão arterial: avaliação e qualificação de estratégias com ênfase na educação nutricional [tese]. São Paulo: Faculdade de Saúde Pública, Universidade de São Paulo; 2011. Disponível em: <http://www.teses.usp.br/teses/disponiveis/6/6138/ tde-27072011-041835/en.php>.

16. Borba AKOT. Diabetes no idoso: práticas educativas e fatores associados à adesão terapêutica [dissertação]. Recife: Universidade Federal de Pernambuco; 2012.

17. Torres HC, Reis IA, Roque C, Faria P. Monitora- 
mento telefônico como estratégia educativa para o autocuidado das pessoas com diabetes na Atenção Primária. Cienc Enferm [Internet]. 2013 [Citado em: 22 nov 2014]; 19(1):95-105. Disponível em: <http://www. scielo.cl $/$ scielo.php? script $=$ sci_arttext\&pid $=$ S0717 $95532013000100009 \& \operatorname{lng}=\mathrm{es}>$.

18. Morgan BS. Avaliação do monitoramento telefônico na promoção do autocuidado em diabetes na atenção primária em saúde [dissertação]. Belo Horizonte: Escola de Enfermagem, Universidade Federal de Minas Gerais; 2013. Disponível em: <http://www.enf.ufmg.br/pos/ defesas/752M.PDF>.

19. Melo LP. Análise biopolítica do discurso oficial sobre educação em saúde para pacientes diabéticos no Brasil. Saúde Soc [internet]. 2013 [citado em 29 nov 2014];22(4):1216-25. Disponível em: <http://www.scielo. br/pdf/sausoc/v22n4/22.pdf>.

20. Torres HC, Hortale VA, Schall V. A experiência de jogos em grupos operativos na educação em saúde para diabéticos. Cad Saúde Pública [Internet]. ago 2003 [citado em 27 nov 2014];19(4):1039-47. Disponível em: <http:/ / www.scielo.br/pdf/csp/v19n4/16853.pdf>.

21. Torres HC, Souza ER, Lima MHM, Bodstein RC. Intervenção educativa para o autocuidado de indíviduos com diabetes mellitus. Acta Paul Enferm. 2011;24(4):51419. Disponível em: <http://dx.doi.org/10.1590/S010321002011000400011>.

22. Vieira G.LC. Avaliação da educação em grupos operativos com usuários diabéticos tipo 2 em Unidades Básicas de Saúde [dissertação]. Belo Horizonte: Escola de Enfermagem, Universidade Federal de Minas Gerais; 2011. Disponível em: < http://www.enf.ufmg.br/site_novo/modules/mastop_publish/files/files_4e71f255f1694.pdf>.

23. Silva RV, Costa PP, Fermino JS. Vivência de educação em saúde: o grupo enquanto proposta de atuação. Trab Educ Saúde [Internet]. 2008 [Citado em: 28 set 2014];6(3). Disponível em: <http://www.scielo.br/scielo.php?script $=$ sci_arttext\&pid $=\mathrm{S} 1981$ $77462008000300014 \& \operatorname{lng}=$ en\&nrm $=$ iso $>$.

24. Moreno DMFC, Visani I, Ruiz MRAR, Muller L, Bittencourt JC, Pelicioni AF. Avaliação das ações de promoção de saúde em hipertensão e diabetes em três Unidades Básicas de Saúde no município de São Paulo [Internet]. 2008 [Citado em: 30 nov 2014];23-5. Disponível em: $\quad<$ http://pesquisa.bvs.br/brasil/resource/pt/sus$26788>$.

25. Matsumoto PM, Barreto ARB, Sakata KN, Siqueira YMC, Zoboli ELCP, Fracolli LA. A educação em saúde no cuidado de usuários do Programa Automonitoramento Glicêmico. Rev esc enferm USP [Internet]. 2012 jun [Citado em: 3 nov 2014];46(3):761-5. Disponível em: <http:// www.scielo.br/scielo.php?script=sci_arttext\&pid=S008062342012000300031\&lng=en $>$.

26. Mendonça FF. Grupos de educação em saúde como espaço de construção de corresponsabilidades: um estudo de caso [tese]. Londrina: Universidade Estadual de Londrina; 2012.

27. Grillo MFF. Avaliação do impacto de um modelo de educação estruturada em grupo para pacientes com diabetes tipo 2 em Atenção Primária [tese]. Porto Alegre: Faculdade de Medicina, Universidade Federal do Rio Grande do Sul; 2011.

28. Rodrigues MTG. Adesão ao tratamento nutricional para o diabetes mellitus em serviço de Atenção Primária à Saúde [dissertação]. Belo Horizonte: Escola de Enfermagem, Universidade Federal de Minas Gerais; 2011.

29. Pereira DA, Costa NMSC, Sousa ALL, Jardim PCBV, Zanini CRO. Efeito de intervenção educativa sobre o conhecimento da doença em pacientes com diabetes mellitus. Rev Latino-Am Enfermagem [Internet]. 2012 jun [Citado em: 24 nov 2014];20(3):478-85. Disponível em: <http:// www.scielo.br/scielo.php?script $=$ sci_arttext\&pid $=$ S010411692012000300008\&lng=en $>$.

30. Torres HC, Roque C, Nunes C. Visita domiciliar: estratégia educativa para o autocuidado de clientes diabéticos na Atenção Básica. Rev Enferm UERJ [Internet]. 2011 jan-mar [Citado em 24 nov 2014];19(1):89-93. Disponível em: <http://www.facenf.uerj.br/v19n1/v19n1a15.pdf>.

31. Torres HC, Santos LM, Cordeiro PMCS. Visita domiciliária: estratégia educativa em saúde para o autocuidado em diabetes. Acta Paul Enferm [Internet]. 2014 fev [Citado em: 24 nov 2014];27(1):23-8. Disponível em: $\quad<$ http://www.scielo.br/scielo.php?script=sci_ arttext\&pid $=$ S0103-21002014000100006\&lng $=$ en. http://dx.doi.org/10.1590/1982-0194201400006>.

32. Guimarães FPM, Takayanagui AMM. Orientações recebidas do serviço de saúde por pacientes para 
o tratamento do portador de diabetes mellitus tipo 2. Rev Nutr [Internet]. 2002 jan [Citado em: 19 nov 2014];15(1):37-44. Disponível em: <http://www. scielo.br/scielo.php?script $=$ sci_arttext\&pid $=\mathrm{S} 1415$ 52732002000100005\&lng=en>.

33. Ministério da Saúde (Brasil). Secretaria de Atenção à Saúde. Departamento de Atenção Básica. Política Nacional de Alimentação e Nutrição. Brasilia: Ministério da Saúde; 2012.

34. Borba AKOT, Marques APO, Leal MCC, Ramos RSPS. Práticas educativas em diabetes mellitus: revisão integrativa da literatura. Rev Gaúcha Enferm [Internet]. 2012 mar [Citado em: 22 nov 2014]; 33(1):169-76. Disponível em: <http://www.scielo.br/scielo.php?script=sci_ arttext\&pid $=$ S1983-14472012000100022\&lng $=$ en $>$.

35. Santos AL. Educação para a saúde de diabéticos através dos círculos de cultura: uma estratégia de intervenção em enfermagem [dissertação]. Maringá: Universidade Estadual de Maringá; 2011.

36. Nunes TTV. Estudo da adesão a um programa de tratamento não farmacológico em idosos com diabetes mellitus tipo 2 [dissertação]. Fortaleza: Universidade de Fortaleza; 2011.

37. Mendes GF. Efeitos da adesão a um programa de educação em diabetes com ênfase em exercícios físicos orientados no controle glicêmico em diabéticos tipo 2 [dissertação]. Brasília: Faculdade de Educação Física, Universidade de Brasília; 2011.

Submissão: dezembro de 2015

Aprovação: novembro de 2016 